\title{
Nonlinear Quantum Neuro-Psycho-Dynamics with Topological Phase Transitions
}

\author{
Vladimir G. Ivancevic* Tijana T. Ivancevic ${ }^{\dagger}$
}

\begin{abstract}
We have proposed a novel model of general quantum, stochastic and chaotic psychodynamics. The model is based on the previously developed Life-Space Foam (LSF) framework to motivational and cognitive dynamics. The present model extends the LSF-approach by incorporating chaotic and topological non-equilibrium phase transitions. Such extended LSF-model is applied for rigorous description of multi-agent joint action. The present model is related to Haken-Kelso-Bunz model of self-organization in the human motor system (including: multi-stability, phase transitions and hysteresis effects, presenting a contrary view to the purely feedback driven neural systems), as well as the entropy-approach to adaptation in human goal-directed motor control.
\end{abstract}

Keywords: Quantum probability, Life-Space Foam, noisy decision making, chaos, topological phase transitions, multi-agent joint action, goal-directed motor control

\footnotetext{
*Human Systems Integration, Land Operations Division, Defence Science \& Technology Organisation, P.O. Box 1500, Edinburgh SA 5111, Australia (Vladimir.Ivancevic@dsto.defence.gov.au)

${ }^{\dagger}$ School of Electrical and Information Engineering, University of South Australia, Mawson Lakes, S.A. 5095, Australia (Tijana.Ivancevic@unisa.edu.au)
} 


\section{Introduction}

Classical physics has provided a strong foundation for understanding brain function through measuring brain activity, modelling the functional connectivity of networks of neurons with algebraic matrices, and modelling the dynamics of neurons and neural populations with sets of coupled differential equations (Freeman, 1975, 2000). Various tools from classical physics enabled recognition and documentation of aspects of the physical states of the brain; the structures and dynamics of neurons, the operations of membranes and organelles that generate and channel electric currents; and the molecular and ionic carriers that implement the neural machineries of electrogenesis and learning. They support description of brain functions at several levels of complexity through measuring neural activity in the brains of animal and human subjects engaged in behavioral exchanges with their environments. One of the key properties of brain dynamics are the coordinated oscillations of populations of neurons that change rapidly in concert with changes in the environment (Freeman and Vitiello, 2006; Ivancevic, 2006a, 2007b). Also, most experimental neurobiologists and neural theorists have focused on sensorimotor functions and their adaptations through various forms of learning and memory. Reliance has been placed on measurements of the rates and intervals of trains of action potentials of small numbers of neurons that are tuned to perceptual invariances and modelling neural interactions with discrete networks of simulated neurons. These and related studies have given a vivid picture of the cortex as a mosaic of modules, each of which performs a sensory or motor function; they have not given a picture of comparable clarity of the integration of modules.

According to Freeman and Vitiello (2006), many-body quantum field theory appears to be the only existing theoretical tool capable to explain the dynamic origin of long-range correlations, their rapid and efficient formation and dissolution, their interim stability in ground states, the multiplicity of coexisting and possibly noninterfering ground states, their degree of ordering, and their rich textures relating to sensory and motor facets of behaviors. It is historical fact that many-body quantum field theory has been devised and constructed in past decades exactly to understand features like ordered pattern formation and phase transitions in condensed matter physics that could not be understood in classical physics, similar to those in the brain.

The domain of validity of the 'quantum' is not restricted to the microscopic world (Umezava, 1993). There are macroscopic features of classically behaving systems, which cannot be explained without recourse to the quantum dynamics. This field theoretic model leads to the view of the phase transition as a condensation that is comparable to the formation of fog and rain drops from water vapor, and that might serve to model both the gamma and beta phase transitions. According to such a model, the production of activity with long-range correlation in the brain takes place through the mechanism of spontaneous breakdown of symmetry (SBS), which has for decades been shown to describe long-range correlation in condensed matter physics. The adoption of such a field theoretic approach enables modelling of the whole cerebral hemisphere and its hierarchy of components down to the atomic level as a fully integrated macroscopic quantum system, namely as a macroscopic system which is a quantum 
system not in the trivial sense that it is made, like all existing matter, by quantum components such as atoms and molecules, but in the sense that some of its macroscopic properties can best be described with recourse to quantum dynamics (see Freeman and Vitiello, 2006 and references therein).

It is well-known that non-equilibrium phase transitions (Haken, 1983, 1993, 1996) are phenomena which bring about qualitative physical changes at the macroscopic level in presence of the same microscopic forces acting among the constituents of a system. Phase transitions can also be associated with autonomous robot competence levels, as informal specifications of desired classes of behaviors for robots over all environments they will encounter, as described by Brooks' subsumption architecture approach (Brooks, 1986, 1989, 1990). The distributed network of augmented finite-state machines can exist in different phases or modalities of their state-space variables, which determine the systems intrinsic behavior. The phase transition represented by this approach is triggered by either internal (a set-point) or external (a command) control stimuli, such as a command to transition from a sleep mode to awake mode, or walking to running.

On the other hand, it is well-known that humans possess more degrees of freedom than are needed to perform any defined motor task, but are required to co-ordinate them in order to reliably accomplish high-level goals, while faced with intense motor variability. In an attempt to explain how this takes place, Todorov and Jordan (2002) formulated an alternative theory of human motor coordination based on the concept of stochastic optimal feedback control. They were able to conciliate the requirement of goal achievement (e.g., grasping an object) with that of motor variability (biomechanical degrees of freedom). Moreover, their theory accommodates the idea that the human motor control mechanism uses internal 'functional synergies' to regulate task-irrelevant (redundant) movement.

Until recently, research concerning sensory processing and research concerning motor control have followed parallel but independent paths. The partitioning of the two lines of research in practice partly derived from and partly fostered a bipartite view of sensorimotor processing in the brain - that a sensory/perceptual system creates a general purpose representation of the world which serves as the input to the motor systems (and other cognitive systems) that generate action/behavior as an output. Recent results from research on vision in natural tasks have seriously challenged this view, suggesting that the visual system does not generate a general-purpose representation of the world, but rather extracts information relevant to the task at hand (Droll et al, 2005; Land and Hayhoe, 2001). At the same time, researchers in motor control have developed an increasing understanding of how sensory limitations and sensory uncertainty can shape the motor strategies that humans employ to perform tasks. Moreover, many aspects of the problem of sensorimotor control are specific to the mapping from sensory signals to motor outputs and do not exist in either domain in isolation. Sensory feedback control of hand movements, coordinate transformations of spatial representations and the influence of processing speed and attention on sensory contributions to motor control are just a few of these. In short, to understand how human (and animal) actors use sensory information to guide motor behavior, we must study sensory and motor systems as an 
integrated whole rather than as decomposable modules in a sequence of discrete processing steps (Knill et al, 2007).

Cognitive neuroscience investigations, including fMRI studies of human co-action, suggest that cognitive and neural processes supporting co-action include joint attention, action observation, task sharing, and action coordination (Fogassi et al, 2005; Knoblich and Jordan, 2003; Newman et al, 2007; Sebanz at al, 2006). For example, when two actors are given a joint control task (e.g., tracking a moving target on screen) and potentially conflicting controls (e.g., one person in charge of acceleration, the other - deceleration), their joint performance depends on how well they can anticipate each other's actions. In particular, better coordination is achieved when individuals receive real-time feedback about the timing of each other's actions (Sebanz at al, 2006).

A developing field in coordination dynamics involves the theory of social coordination, which attempts to relate the DC to normal human development of complex social cues following certain patterns of interaction. This work is aimed at understanding how human social interaction is mediated by meta-stability of neural networks. fMRI and EEG are particularly useful in mapping thalamocortical response to social cues in experimental studies. In particular, a new theory called the Phi complex has been developed by S. Kelso and collaborators, to provide experimental results for the theory of social coordination dynamics (see the recent nonlinear dynamics paper discussing social coordination and EEG dynamics of Tognoli et al, 2007). According to this theory, a pair of phi rhythms, likely generated in the mirror neuron system, is the hallmark of human social coordination. Using a dual-EEG recording system, the authors monitored the interactions of eight pairs of subjects as they moved their fingers with and without a view of the other individual in the pair.

Recently developed Life Space Foam (LSF) model (Ivancevic and Aidman, 2007) is an integration of two modern approaches to cognition: (i) dynamical field theory (DFT, Amari, 1977; Schöner, 2007) and (ii) quantum-probabilistic dynamics (QP, Glimcher, 2005; Busemayer et al, 2006). In this paper we expand the LSFconcept to model decision making process in human-robot joint action and related LSF-phase transitions.

\section{Classical versus Quantum Probability}

As quantum probability in human cognition and decision making has recently become popular, let us briefly describe this fundamental concept (for more details, see Ivancevic, 2007a, 2007c, 2008b).

\subsection{Classical Probability and Stochastic Dynamics}

Recall that a random variable $X$ is defined by its distribution function $f(x)$. Its probabilistic description is based on the following rules: (i) $P\left(X=x_{i}\right)$ is the probability that $X=x_{i}$; and (ii) $P(a \leq X \leq b)$ is the probability that $X$ lies in a closed interval $[a, b]$. Its statistical description is based on: (i) $\mu_{X}$ or $E(X)$ is the mean or expectation of $X$; and (ii) $\sigma_{X}$ is the standard deviation of $X$. There are two cases of random variables: discrete and continuous, each having its own probability (and statistics) theory. 
A discrete random variable $X$ has only a countable number of values $\left\{x_{i}\right\}$. Its distribution function $f\left(x_{i}\right)$ has the following properties:

$$
\begin{aligned}
& P\left(X=x_{i}\right)=f\left(x_{i}\right), \quad f\left(x_{i}\right) \geq 0, \\
& \sum_{i} f\left(x_{i}\right) d x=1 .
\end{aligned}
$$

Statistical description of $X$ is based on its discrete mean value $\mu_{X}$ and standard deviation $\sigma_{X}$, given respectively by

$$
\begin{aligned}
\mu_{X} & =E(X)=\sum_{i} x_{i} f\left(x_{i}\right), \\
\sigma_{X} & =\sqrt{E\left(X^{2}\right)-\mu_{X}^{2}} .
\end{aligned}
$$

Here $f(x)$ is a piecewise continuous function such that:

$$
\begin{aligned}
& P(a \leq X \leq b)=\int_{a}^{b} f(x) d x, \quad f(x) \geq 0, \\
& \int_{-\infty}^{\infty} f(x) d x=\int_{\mathbb{R}} f(x) d x=1 .
\end{aligned}
$$

Statistical description of $X$ is based on its continuous mean $\mu_{X}$ and standard deviation $\sigma_{X}$, given respectively by

$$
\begin{aligned}
\mu_{X} & =E(X)=\int_{-\infty}^{\infty} x f(x) d x, \\
\sigma_{X} & =\sqrt{E\left(X^{2}\right)-\mu_{X}^{2}} .
\end{aligned}
$$

Now, let us observe the similarity between the two descriptions. The same kind of similarity between discrete and continuous quantum spectrum stroke P. Dirac when he suggested the combined integral approach, that he denoted by $\mathfrak{f}$ - meaning 'both integral and sum at once': summing over a discrete spectrum and integration over a continuous spectrum.
To emphasize this similarity even further, as well as to set-up the stage for the path integral, recall the notion of a cumulative distribution function of a random variable $X$, that is a function $F: \mathbb{R} \rightarrow \mathbb{R}$, defined by

$$
F(a)=P(X) \leq a .
$$

In particular, suppose that $f(x)$ is the distribution function of $X$. Then

$$
\begin{array}{ll}
F(x)=\sum_{x_{i} \leq x} f\left(x_{i}\right), & \text { or } \\
F(x) & =\int_{-\infty}^{\infty} f(t) d t,
\end{array}
$$

according to as $x$ is a discrete or continuous random variable. In either case, $F(a) \leq F(b)$ whenever $a \leq b$. Also,

$\lim _{x \rightarrow-\infty} F(x)=0 \quad$ and $\quad \lim _{x \rightarrow \infty} F(x)=1$, that is, $F(x)$ is monotonic and its limit to the left is 0 and the limit to the right is 1 . Furthermore, its cumulative probability is given by

$$
P(a \leq X \leq b)=F(b)-F(a),
$$

and the Fundamental Theorem of Calculus tells us that, in the continuum case,

$$
f(x)=\partial_{x} F(x) .
$$

Now, recall that Markov stochastic process is a random process characterized by a lack of memory, i.e., the statistical properties of the immediate future are uniquely determined by the present, regardless of the past (Gardiner, 1985; Ivancevic, 2006b).

For example, a random walk is an example of the Markov chain, i.e., a discrete-time Markov process, such that the motion of the 
system in consideration is viewed as a sequence of states, in which the transition from one state to another depends only on the preceding one, or the probability of the system being in state $k$ depends only on the previous state $k-1$. The property of a Markov chain of prime importance in biomechanics is the existence of an invariant distribution of states: we start with an initial state $x_{0}$ whose absolute probability is 1. Ultimately the states should be distributed according to a specified distribution.

Between the pure deterministic dynamics, in which all DOF of the system in consideration are explicitly taken into account, leading to classical dynamical equations, for example in Hamiltonian form (using $\partial_{x} \equiv \partial / \partial x$ ),

$$
\dot{q}^{i}=\partial_{p_{i}} H, \quad \dot{p}_{i}=-\partial_{q^{i}} H,
$$

(where $q^{i}, p_{i}$ are coordinates and momenta, while $H=H(q, p)$ is the total system energy) - and pure stochastic dynamics (Markov process), ther is so-called hybrid dynamics, particularly Brownian dynamics, in which some of DOF are represented only through their stochastic influence on others. As an example, suppose a system of particles interacts with a viscous medium. Instead of specifying a detailed interaction of each particle with the particles of the viscous medium, we represent the medium as a stochastic force acting on the particle. The stochastic force reduces the dimensionally of the dynamics.

Recall that the Brownian dynamics represents the phase-space trajectories of a collection of particles that individually obey Langevin rate equations in the field of force (i.e., the particles interact with each other via some deterministic force). For a free particle, the Langevin equation reads (Gardiner, 1985):

$$
m \dot{v}=R(t)-\beta v,
$$

where $m$ denotes the mass of the particle and $v$ its velocity. The right-hand side represent the coupling to a heat bath; the effect of the random force $R(t)$ is to heat the particle. To balance overheating (on the average), the particle is subjected to friction $\beta$. In humanoid dynamics this is performed with the RayleighVan der Pol's dissipation. Formally, the solution to the Langevin equation can be written as

$$
\begin{aligned}
& v(t)=v(0) \exp \left(-\frac{\beta}{m} t\right) \\
& +\frac{1}{m} \int_{0}^{t} \exp [-(t-\tau) \beta / m] R(\tau) d \tau,
\end{aligned}
$$

where the integral on the right-hand side is a stochastic integral and the solution $v(t)$ is a random variable. The stochastic properties of the solution depend significantly on the stochastic properties of the random force $R(t)$. In the Brownian dynamics the random force $R(t)$ is Gaussian distributed. Then the problem boils down to finding the solution to the Langevin stochastic differential equation with the supplementary condition (zero and mean variance)

$<R(t)>=0, \quad<R(t) R(0)>=2 \beta k_{B} T \delta(t)$, where $<$. $>$ denotes the mean value, $T$ is temperature, $k_{B}$-equipartition (i.e., uniform distribution of energy) coefficient, Dirac $\delta(t)$-function.

Algorithm for computer simulation of the Brownian dynamics (for a single particle) can be written as (Heermann, 1990):

1. Assign an initial position and velocity. 
2. Draw a random number from a Gaussian distribution with mean zero and variance.

3. Integrate the velocity to get $v^{n+1}$.

4. Add the random component to the velocity.

Another approach to taking account the coupling of the system to a heat bath is to subject the particles to collisions with virtual particles (Heermann, 1990). Such collisions are imagined to affect only momenta of the particles, hence they affect the kinetic energy and introduce fluctuations in the total energy. Each stochastic collision is assumed to be an instantaneous event affecting only one particle.

The collision-coupling idea is incorporated into the Hamiltonian model of dynamics (1) by adding a stochastic force $R_{i}=R_{i}(t)$ to the $\dot{p}$ equation

$$
\dot{q}^{i}=\partial_{p_{i}} H, \quad \dot{p}_{i}=-\partial_{q^{i}} H+R_{i}(t) .
$$

On the other hand, the so-called Ito stochastic integral represents a kind of classical RiemannStieltjes integral from linear functional analysis, which is (in 1D case) for an arbitrary timefunction $G(t)$ defined as the mean square limit

$$
\begin{aligned}
& \int_{t_{0}}^{t} G(t) d W(t)= \\
& m s \lim _{n \rightarrow \infty}\left\{\sum _ { i = 1 } ^ { n } G \left(t_{i-1}\left[W\left(t_{i}\right)-W\left(t_{i-1}\right]\right\} .\right.\right.
\end{aligned}
$$

Now, the general ND Markov process can be defined by Ito stochastic differential equation (SDE),

$$
\begin{aligned}
d x_{i}(t) & =A_{i}\left[x^{i}(t), t\right] d t+B_{i j}\left[x^{i}(t), t\right] d W^{j}(t), \\
x^{i}(0) & =x_{i 0}, \quad(i, j=1, \ldots, N)
\end{aligned}
$$

or corresponding Ito stochastic integral equation

$$
\begin{aligned}
& x^{i}(t)=x^{i}(0)+\int_{0}^{t} d s A_{i}\left[x^{i}(s), s\right] \\
& +\int_{0}^{t} d W^{j}(s) B_{i j}\left[x^{i}(s), s\right]
\end{aligned}
$$

in which $x^{i}(t)$ is the variable of interest, the vector $A_{i}[x(t), t]$ denotes deterministic drift, the matrix $B_{i j}[x(t), t]$ represents continuous stochastic diffusion fluctuations, and $W^{j}(t)$ is an $N-$ variable Wiener process (i.e., generalized Brownian motion, see Wiener, 1961) and

$$
d W^{j}(t)=W^{j}(t+d t)-W^{j}(t) .
$$

Now, there are three well-known special cases of the Chapman-Kolmogorov equation (see Gardiner, 1985):

1. When both $B_{i j}[x(t), t]$ and $W(t)$ are zero, i.e., in the case of pure deterministic motion, it reduces to the Liouville equation

$$
\begin{aligned}
& \partial_{t} P\left(x^{\prime}, t^{\prime} \mid x^{\prime \prime}, t^{\prime \prime}\right)= \\
& -\sum_{i} \frac{\partial}{\partial x^{i}}\left\{A_{i}[x(t), t] P\left(x^{\prime}, t^{\prime} \mid x^{\prime \prime}, t^{\prime \prime}\right)\right\} .
\end{aligned}
$$

2. When only $W(t)$ is zero, it reduces to the Fokker-Planck equation

$$
\begin{gathered}
\partial_{t} P\left(x^{\prime}, t^{\prime} \mid x^{\prime \prime}, t^{\prime \prime}\right)= \\
-\sum_{i} \frac{\partial}{\partial x^{i}}\left\{A_{i}[x(t), t] P\left(x^{\prime}, t^{\prime} \mid x^{\prime \prime}, t^{\prime \prime}\right)\right\}+ \\
\frac{1}{2} \sum_{i j} \frac{\partial^{2}}{\partial x^{i} \partial x^{j}}\left\{B_{i j}[x(t), t] P\left(x^{\prime}, t^{\prime} \mid x^{\prime \prime}, t^{\prime \prime}\right)\right\} .
\end{gathered}
$$

3. When both $A_{i}[x(t), t]$ and $B_{i j}[x(t), t]$ are zero, i.e., the state-space consists of inte- 
gers only, it reduces to the Master equation of discontinuous jumps

$$
\begin{aligned}
& \partial_{t} P\left(x^{\prime}, t^{\prime} \mid x^{\prime \prime}, t^{\prime \prime}\right)= \\
& \int d x W\left(x^{\prime} \mid x^{\prime \prime}, t\right) P\left(x^{\prime}, t^{\prime} \mid x^{\prime \prime}, t^{\prime \prime}\right) \\
& -\int d x W\left(x^{\prime \prime} \mid x^{\prime}, t\right) P\left(x^{\prime}, t^{\prime} \mid x^{\prime \prime}, t^{\prime \prime}\right) .
\end{aligned}
$$

The Markov assumption can now be formulated in terms of the conditional probabilities $P\left(x^{i}, t_{i}\right)$ : if the times $t_{i}$ increase from right to left, the conditional probability is determined entirely by the knowledge of the most recent condition. Markov process is generated by a set of conditional probabilities whose probabilitydensity $P=P\left(x^{\prime}, t^{\prime} \mid x^{\prime \prime}, t^{\prime \prime}\right)$ evolution obeys the general Chapman-Kolmogorov integro-differential equation

$$
\begin{aligned}
& \partial_{t} P=-\sum_{i} \frac{\partial}{\partial x^{i}}\left\{A_{i}[x(t), t] P\right\} \\
& +\frac{1}{2} \sum_{i j} \frac{\partial^{2}}{\partial x^{i} \partial x^{j}}\left\{B_{i j}[x(t), t] P\right\} \\
& +\int d x\left\{W\left(x^{\prime} \mid x^{\prime \prime}, t\right) P-W\left(x^{\prime \prime} \mid x^{\prime}, t\right) P\right\}
\end{aligned}
$$

including deterministic drift, diffusion fluctuations and discontinuous jumps (given respectively in the first, second and third terms on the r.h.s.).

It is this general Chapman-Kolmogorov integro-differential equation, with its conditional probability density evolution, $P=P\left(x^{\prime}, t^{\prime} \mid x^{\prime \prime}, t^{\prime \prime}\right)$ that we are going to model by the Feynman path integral $£$, providing us with the physical insight behind the abstract (conditional) probability densities.

\subsection{Quantum Probability Concept}

An alternative concept of probability, the socalled quantum probability, is based on the following physical facts (elaborated in detail in this section):

1. The time-dependent Schrödinger equation represents a complex-valued generalization of the real-valued Fokker-Planck equation for describing the spatio-temporal probability density function for the system exhibiting continuous-time Markov stochastic process.

2. The Feynman path integral $£$ is a generalization of the time-dependent Schrödinger equation, including both continuoustime and discrete-time Markov stochastic processes.

3. Both Schrödinger equation and path integral give 'physical description' of any system they are modelling in terms of its physical energy, instead of an abstract probabilistic description of the FokkerPlanck equation.

Therefore, the Feynman path integral $£$, as a generalization of the time-dependent Schrödinger equation, gives a unique physical description for the general Markov stochastic process, in terms of the physically based generalized probability density functions, valid both for continuous-time and discrete-time Markov 'systems.

Basic consequence: a different way for calculating probabilities. The difference is rooted in the fact that sum of squares is different from the square of sums, as is explained in the following text. 
In Dirac-Feynman quantum formalism, each possible route from the initial system state $A$ to the final system state $B$ is called a history. This history comprises any kind of a route (see Figure 1), ranging from continuous and smooth deterministic (mechanical-like) paths to completely discontinues and random Markov chains (see e.g., Gardiner, 1985). Each history (labelled by index $k$ ) is quantitatively described by a complex number, $z_{k}=r_{k} \mathrm{e}^{\mathrm{i} \theta_{k}}$ (with $\mathrm{i}=$ $\sqrt{-1}$ ), called the 'individual transition amplitude'. Its absolute square, $\left|z_{k}\right|^{2}$, is called the individual transition probability. Now, the total transition amplitude is the sum of all individual transition amplitudes, $\sum_{k} z_{k}$, called the sum-over-histo-ries. The absolute square of this sum-over-histories, $\left|\sum_{k} z_{k}\right|^{2}$, is the total transition probability.

In this way, the overall probability of the system's transition from some initial state $A$ to some final state $B$ is given not by adding up the probabilities for each history-route, but by 'head-to-tail' adding up the sequence of amplitudes making-up each route first (i.e., performing the sum-over-histories) - to get the total amplitude as a 'resultant vector', and then squaring the total amplitude to get the overall transition probability.

\section{The Life Space Foam}

General nonlinear attractor dynamics, both deterministic and stochastic, as well as possibly chaotic, developed in the framework of Feynman path integrals, have recently been applied by Ivancevic and Aidman (2007) to formalize classical Lewinian field-theoretic psychodynamics (Lewin, 1951, 1997; Gold, 1999), resulting in the development of a new concept of life-space

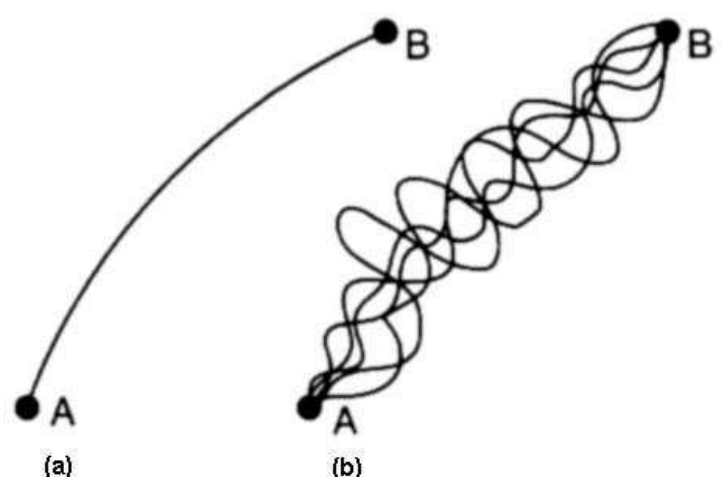

(a)

(b)

Figure 1: Two ways of physical transition from an initial state $A$ to the corresponding final state $B$. (a) Classical physics proposes a single deterministic trajectory, minimizing the total system's energy. (b) Quantum physics proposes a family of Markov stochastic histories, namely all possible routes from $A$ to $B$, both continuous-time and discrete-time Markov chains, each giving an equal contribution to the total transition probability.

foam (LSF) as a natural medium for motivational and cognitive psychodynamics. According to the LSF-formalism, the classic Lewinian life space can be macroscopically represented as a smooth manifold with steady force-fields and behavioral paths, while at the microscopic level it is more realistically represented as a collection of wildly fluctuating force-fields, (loco)motion paths and local geometries (and topologies with holes).

We have used the new LSF concept to develop modelling framework for motivational dynamics (MD) and induced cognitive dynamics (CD). Motivation processes both precede and coincide with every goal-directed action. Usually these motivation processes include the sequence of the following four feedforward phases (Ivancevic and Aidman, 2007): (*) 
1. Intention Formation $\mathcal{F}$, including: decision making, commitment building, etc.

2. Action Initiation $\mathcal{I}$, including: handling conflict of motives, resistance to alternatives, etc.

3. Maintaining the Action $\mathcal{M}$, including: resistance to fatigue, distractions, etc.

4. Termination $\mathcal{T}$, including parking and avoiding addiction, i.e., staying in control.

With each of the phases $\{\mathcal{F}, \mathcal{I}, \mathcal{M}, \mathcal{T}\}$ in $(*)$, we can associate a transition propagator - an ensemble of (possibly crossing) feedforward paths propagating through the 'wood of obstacles' (including topological holes in the LSF, see Figure 3), so that the complete transition is a product of propagators (as well as sum over paths). All the phases-propagators are controlled by a unique Monitor feedback process.

A set of least-action principles is used to model the smoothness of global, macro-level LSF paths, fields and geometry, according to the following prescription. The action $S[\Phi]$, psycho-physical dimensions of

$$
\text { Energy } \times \text { Time }=\text { Effort }
$$

and depending on macroscopic paths, fields and geometries (commonly denoted by an abstract field symbol $\left.\Phi^{i}\right)$ is defined as a temporal integral from the initial time instant $t_{i n i}$ to the final time instant $t_{f i n}$,

$$
S[\Phi]=\int_{t_{i n i}}^{t_{f i n}} \mathfrak{L}[\Phi] d t
$$

with Lagrangian density given by

$$
\mathfrak{L}[\Phi]=\int d^{n} x \mathcal{L}\left(\Phi_{i}, \partial_{x^{j}} \Phi^{i}\right),
$$

where the integral is taken over all $n$ coordinates $x^{j}=x^{j}(t)$ of the LSF, and $\partial_{x^{j}} \Phi^{i}$ are time and space partial derivatives of the $\Phi^{i}$-variables over coordinates. The standard least action principle

$$
\delta S[\Phi]=0,
$$

gives, in the form of the so-called Euler-Lagrangian equations, a shortest (loco)motion path, an extreme force-field, and a life-space geometry of minimal curvature (and without holes). In this way, we effectively derive a unique globally smooth transition map

$F: I N T E N T I O N_{t_{i n i}} \longrightarrow A C T I O N_{t_{f i n}}$,

performed at a macroscopic (global) time-level from some initial time $t_{i n i}$ to the final time $t_{f i n}$. In this way, we have obtained macroobjects in the global LSF: a single path described by Newtonian-like equation of motion, a single force-field described by Maxwellianlike field equations, and a single obstacle-free Riemannian geometry (with global topology without holes).

To model the corresponding local, microlevel LSF structures of rapidly fluctuating cognitive dynamics, an adaptive path integral is formulated, defining a multi-phase and multipath (multi-field and multi-geometry) transition amplitude from the state of Intention to the state of Action,

$$
\langle\text { Action }| \text { Intention }\rangle_{\text {total }}:=£ \mathcal{D}[w \Phi] \mathrm{e}^{\mathrm{i} S[\Phi]},
$$

where the Lebesgue integration is performed over all continuous $\Phi_{\text {con }}^{i}=$ paths + fields + geometries, while summation is performed over all discrete processes and regional topologies $\Phi_{\text {dis }}^{j}$. The symbolic differential $\mathcal{D}[w \Phi]$ in the 
general path integral (5), represents an adaptive path measure, defined as a weighted product (with $i=1, \ldots, n=$ con + dis)

$$
\mathcal{D}[w \Phi]=\lim _{N \longrightarrow \infty} \prod_{s=1}^{N} w_{s} d \Phi_{s}^{i} .
$$

The adaptive path integral (5) -(6) represents an $\infty$-dimensional neural network, with weights $w$ updating by the general rule (Ivancevic and Aidman, 2007):

new value $(t+1)=$ old value $(t)+$ innovation $(t)$.

The adaptive path integral (5) incorporates the local Bernstein adaptation process (Bernstein, 1967, 1982):

$$
\text { desired state } S W(t+1)=
$$

current state $I W(t)+$ adjustment step $\Delta W(t)$

as well as the augmented finite state machine of Brooks' subsumption architecture (Brooks, 1986, 1989, 1990), with a networked behavior function:

$$
\begin{aligned}
& \text { final state } w(t+1)= \\
& \text { current state } w(t)+ \\
& \text { adjustment behavior } f(\Delta w(t)) .
\end{aligned}
$$

We remark here that the traditional neural networks approaches are known for their classes of functions they can represent. This limitation has been attributed to their low-dimensionality (the largest neural networks are limited to the order of $10^{5}$ dimensions, see Izhikevich and Edelman, 2008). The proposed path integral approach represents a new family of function-representation methods, which potentially offers a basis for a fundamentally more expansive solution.
On the macro-level in $L S F$ we have the (loco)motion action principle

$$
\delta S[x]=0,
$$

with the Newtonian-like action $S[x]$ given by

$$
S[x]=\int_{t_{\text {ini }}}^{t_{f i n}} d t\left[\frac{1}{2} g_{i j} \dot{x}^{i} \dot{x}^{j}+\varphi^{i}\left(x^{i}\right)\right],
$$

where $\dot{x}^{i}$ represents motivational (loco)motion velocity vector with cognitive processing speed. The first bracket term in (77) represents the kinetic energy $T$,

$$
T=\frac{1}{2} g_{i j} \dot{x}^{i} \dot{x}^{j},
$$

generated by the Riemannian metric tensor $g_{i j}$, while the second bracket term, $\varphi^{i}\left(x^{i}\right)$, denotes the family of potential force-fields, driving the (loco)motions $x^{i}=x^{i}(t)$ (the strengths of the fields $\varphi^{i}\left(x^{i}\right)$ depend on their positions $x^{i}$ in LSF. The corresponding Euler-Lagrangian equation gives the Newtonian-like equation of motion

$$
\frac{d}{d t} T_{\dot{x}^{i}}-T_{x^{i}}=-\varphi_{x^{i}}^{i}
$$

(subscripts denote the partial derivatives), which can be put into the standard Lagrangian form

$$
\frac{d}{d t} L_{\dot{x}^{i}}=L_{x^{i}}, \quad \text { with } \quad L=T-\varphi^{i}\left(x^{i}\right) .
$$

Now, according to Lewin, the life space also has a sophisticated topological structure. As a Riemannian smooth $n$-manifold, the LSFmanifold $\boldsymbol{\Sigma}$ gives rise to its fundamental $n-$ groupoid, or $n$-category $\Pi_{n}(\Sigma)$ (see Ivancevic, 2006b, 2007a). In $\Pi_{n}(\Sigma)$, 0-cells are points in $\boldsymbol{\Sigma}$; 1 -cells are paths in $\boldsymbol{\Sigma}$ (i.e., parameterized smooth maps $f:[0,1] \rightarrow \Sigma) ; 2$-cells are smooth homotopies (denoted by $\simeq$ ) of paths relative 
to endpoints (i.e., parameterized smooth maps $h:[0,1] \times[0,1] \rightarrow \Sigma) ; 3$-cells are smooth homotopies of homotopies of paths in $\boldsymbol{\Sigma}$ (i.e., parameterized smooth maps $j:[0,1] \times[0,1] \times[0,1] \rightarrow$ $\Sigma)$. Categorical composition is defined by pasting paths and homotopies. In this way, the following recursive homotopy dynamics emerges on the LSF-manifold $\boldsymbol{\Sigma}(* *)$ : 
$0-$ cell $: x_{0} \bullet \quad x_{0} \in M ; \quad$ in the higher cells below: $t, s \in[0,1]$;

$1-\operatorname{cell}: x_{0} \bullet \stackrel{f}{\longrightarrow} \bullet x_{1} \quad f: x_{0} \simeq x_{1} \in M$,

$f:[0,1] \rightarrow M, f: x_{0} \mapsto x_{1}, x_{1}=f\left(x_{0}\right), f(0)=x_{0}, f(1)=x_{1} ;$

e.g., linear path: $f(t)=(1-t) x_{0}+t x_{1}$; $\quad$ or

Euler-Lagrangian $f$-dynamics with endpoint conditions $\left(x_{0}, x_{1}\right)$ :

$\frac{d}{d t} f_{\dot{x}^{i}}=f_{x^{i}}, \quad$ with $\quad x(0)=x_{0}, \quad x(1)=x_{1}, \quad(i=1, \ldots, n) ;$

$2-\operatorname{cell}: x_{0} \bullet \overbrace{g}^{f} \bullet x_{1} \quad h: f \simeq g \in M$,

$h:[0,1] \times[0,1] \rightarrow M, h: f \mapsto g, g=h\left(f\left(x_{0}\right)\right)$,

$h\left(x_{0}, 0\right)=f\left(x_{0}\right), h\left(x_{0}, 1\right)=g\left(x_{0}\right), h(0, t)=x_{0}, h(1, t)=x_{1}$

e.g., linear homotopy: $h\left(x_{0}, t\right)=(1-t) f\left(x_{0}\right)+t g\left(x_{0}\right)$; $\quad$ or

homotopy between two Euler-Lagrangian $(f, g)$ - dynamics

with the same endpoint conditions $\left(x_{0}, x_{1}\right)$ :

$\frac{d}{d t} f_{\dot{x}^{i}}=f_{x^{i}}, \quad$ and $\quad \frac{d}{d t} g_{\dot{x}^{i}}=g_{x^{i}} \quad$ with $\quad x(0)=x_{0}, \quad x(1)=x_{1} ;$

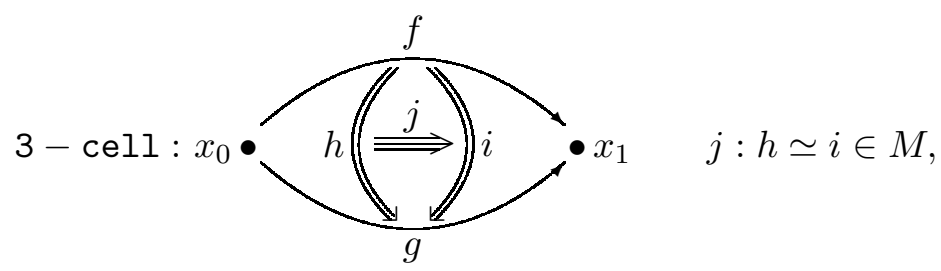

$j:[0,1] \times[0,1] \times[0,1] \rightarrow M, j: h \mapsto i, i=j\left(h\left(f\left(x_{0}\right)\right)\right)$

$j\left(x_{0}, t, 0\right)=h\left(f\left(x_{0}\right)\right), j\left(x_{0}, t, 1\right)=i\left(f\left(x_{0}\right)\right)$,

$j\left(x_{0}, 0, s\right)=f\left(x_{0}\right), j\left(x_{0}, 1, s\right)=g\left(x_{0}\right)$,

$j(0, t, s)=x_{0}, j(1, t, s)=x_{1}$

e.g., linear composite homotopy: $j\left(x_{0}, t, s\right)=(1-t) h\left(f\left(x_{0}\right)\right)+t i\left(f\left(x_{0}\right)\right)$;

or, homotopy between two homotopies between above two Euler-

Lagrangian $(f, g)$ - dynamics with the same endpoint conditions $\left(x_{0}, x_{1}\right)$. 
On the micro-LSF level, instead of a single path defined by the Newtonian-like equation of motion (8), we have an ensemble of fluctuating and crossing paths with weighted probabilities (of the unit total sum). This ensemble of micro-paths is defined by the simplest instance of our adaptive path integral (5), similar to the Feynman's original sum over histories,

$$
\langle\text { Action }| \text { Intention }\rangle_{\text {paths }}=£ \mathcal{D}[w x] \mathrm{e}^{\mathrm{i} S[x]},
$$

where $\mathcal{D}[w x]$ is a functional measure on the space of all weighted paths, and the exponential depends on the action $S[x]$ given by (7). This procedure can be redefined in a mathematically cleaner way if we Wick-rotate the time variable $t$ to imaginary values, $t \mapsto \tau=\mathrm{i} t$, thereby making all integrals real:

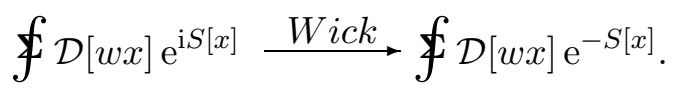

Discretization of (10) gives the standard thermodynamic-like partition function

$$
Z=\sum_{j} \mathrm{e}^{-w_{j} E^{j} / T}
$$

where $E^{j}$ is the motion energy eigenvalue (reflecting each possible motivational energetic state $T$ is the temperature-like environmental control parameter, and the sum runs over all motion energy eigenstates (labelled by the index $j$ ). From (11), we can further calculate all thermodynamic-like and statistical properties of $\mathrm{MD}$ and $\mathrm{CD}$, as for example, transition entropy, $S=k_{B} \ln Z$, etc.

\subsection{Noisy Decision Making in the LSF}

From CD-perspective, our adaptive path integral (9) calculates all (alternative) pathways of information flow during the transition Intention $\rightarrow$ Action. In the connectionist language, (9) represents activation dynamics, to which our Monitor process gives a kind of backpropagation feedback, a common type of supervised learning 1

$$
w_{s}(t+1)=w_{s}(t)-\eta \nabla J(t),
$$

where $\eta$ is a small constant, called the step size, or the learning rate, and $\nabla J(n)$ denotes the gradient of the 'performance hyper-surface' at the $t$-th iteration.

Now, the basic question about our local decision making process, occurring under uncertainty at the intention formation faze $\mathcal{F}$, is: Which alternative to choose? In our pathintegral language this reads: Which path (alternative) should be given the highest probability weight $w$ ? This problem can be either iteratively solved by the learning process (12), controlled by the MONITOR feedback, which we term algorithmic approach, or by the local decision making process under uncertainty, which we term heuristic approach (Ivancevic and Aidman, 2007). This qualitative analysis is based on the micro-level interpretation of the Newtonian-like action $S[x]$, given by (77) and figuring both processing speed $\dot{x}$ and LTM (i.e., the force-field $\varphi(x)$, see next subsection). Here we consider three different cases:

1. If the potential $\varphi(x)$ is not very dependent upon position $x(t)$, then the more direct paths contribute the most, as longer

\footnotetext{
${ }^{1}$ Note that we could also use a reward-based, reinforcement learning rule (Suttton and Barto, 1998), in which system learns its optimal policy:
} 
paths, with higher mean square velocities $[\dot{x}(t)]^{2}$ make the exponent more negative (after Wick rotation (10)).

2. On the other hand, suppose that $\varphi(x)$ does indeed depend on position $x$. For simplicity, let the potential increase for the larger values of $x$. Then a direct path does not necessarily give the largest contribution to the overall transition probability, because the integrated value of the potential is higher than over another paths.

3. Finally, consider a path that deviates widely from the direct path. Then $\varphi(x)$ decreases over that path, but at the same time the velocity $\dot{x}$ increases. In this case, we expect that the increased velocity $\dot{x}$ would more than compensate for the decreased potential over the path.

Therefore, the most important path (i.e., the path with the highest weight $w$ ) would be the one for which any smaller integrated value of the surrounding field potential $\varphi(x)$ is more than compensated for by an increase in kineticlike energy $\frac{m}{2} \dot{x}^{2}$. In principle, this is neither the most direct path, nor the longest path, but rather a middle way between the two. Formally, it is the path along which the average Lagrangian is minimal,

$$
<\frac{m}{2} \dot{x}^{2}+\varphi(x)>\longrightarrow \min ,
$$

i.e., the path that requires minimal memory (both LTM and WM) and processing speed. This mechanical result is consistent with the "cognitive filter theory' of selective attention (Broadbent, 1958), which postulates a low level filter that allows only a limited number of percepts to reach the brain at any time. In this theory, the importance of conscious, directed attention is minimized. The type of attention involving low level filtering corresponds to the concept of early selection.

Although we termed this 'heuristic approach' in the sense that we can instantly feel both the processing speed $\dot{x}$ and the LTM field $\varphi(x)$ involved, there is clearly a psycho-physical rule in the background, namely the averaging minimum relation (13).

From the decision making point of view, all possible paths (alternatives) represent the consequences of decision making. They are, by default, short-term consequences, as they are modelled in the micro-time-level. However, the path integral formalism allows calculation of the long-term consequences, just by extending the integration time, $t_{\text {fin }} \rightarrow \infty$. Besides, this averaging decision mechanics - choosing the optimal path - actually performs the 'averaging lift' in the LSF: from the micro-level to the macro-level.

For example, one of the simplest types of performance-degrading disturbances in the LSF is what we term motivational fatigue - a motivational drag factor that slows the actors' progress towards their goal. There are two fundamentally different sources of this motivational drag, both leading to apparently the same reduction in performance: (a) tiredness / exhaustion and (b) satiation (e.g., boredom). Both involve the same underlying mechanism (the raising valence of the alternatives to continuing the action) but the alternatives will differ considerably, depending on the properties of the task, from self-preservation / recuperation in the exhaustion case through to competing goals in the satiation case.

The spatial representation of this motiva- 
tional drag is relatively simple: uni-dimensional LSF-coordinates may be sufficient for most purposes, which makes it attractive for the initial validation of our predictive model. Similarly uncomplicated spatial representations can be achieved for what we term motivational boost derived from the proximity to the goal (including the well-known phenomenon of 'the home stretch'): the closer the goal (e.g., a finishing line) is perceived to be, the stronger its 'pulling power' (Lewin 1951, 1997). Combinations of motivational drag and motivational boost effects may be of particular interest in a range of applications. These combinations can be modelled within relatively simple uni-dimensional LSF-coordinate systems.

\section{Geometric Chaos and Topo- logical Phase Transitions}

In this section we extend the LSF-formalism to incorporate geometrical chaos (Ivancevic et al, 2008; Ivancevic, 2006c, 2008a) and associated topological phase transitions.

It is well-known that on the basis of the ergodic hypothesis, statistical mechanics describes the physics of many-degrees of freedom systems by replacing time averages of the relevant observables with ensemble averages. Therefore, instead of using statistical ensembles, we can investigate the Hamiltonian (microscopic) dynamics of a system undergoing a phase transition. The reason for tackling dynamics is twofold. First, there are observables, like Lyapunov exponents, that are intrinsically dynamical. Second, the geometrization of Hamiltonian dynamics in terms of Riemannian geometry provides new observables and, in general, an interesting framework to investigate the phe- nomenon of phase transitions (Caiani et al, 1997; Pettini, 2007). The geometrical formulation of the dynamics of conservative systems (see Ivancevic, 2006b, 2008a) was first used by Krylov (1979) in his studies on the dynamical foundations of statistical mechanics and subsequently became a standard tool to study abstract systems in ergodic theory.

The simplest, mechanical-like LSF-action in the individual's LSF-manifold $\boldsymbol{\Sigma}$ has a Riemannian locomotion form (summation convention is always assumed)

$$
S[q]=\frac{1}{2} \int_{t_{i n i}}^{t_{f i n}}\left[a_{i j} \dot{q}^{i} \dot{q}^{j}-V(q)\right] d t,
$$

where $a_{i j}$ is the 'material' metric tensor that generates the total 'kinetic energy' of cognitive (loco)motions defined by their configuration coordinates $q^{i}$ and velocities $\dot{q}^{i}$, with the motivational potential energy $V(q)$ and the standard Hamiltonian

$$
H(p, q)=\sum_{i=1}^{N} \frac{1}{2} p_{i}^{2}+V(q),
$$

where $p_{i}$ are the canonical (loco)motion momenta.

Dynamics of $N$ DOF mechanical-like systems with action (14) and Hamiltonian (15) are commonly given by the set of geodesic equations (Ivancevic, 2006b, 2007a)

$$
\frac{d^{2} q^{i}}{d s^{2}}+\Gamma_{j k}^{i} \frac{d q^{j}}{d s} \frac{d q^{k}}{d s}=0
$$

where $\Gamma_{j k}^{i}$ are the Christoffel symbols of the affine Levi-Civita connection of the Riemannian LSF-manifold $\boldsymbol{\Sigma}$.

Alternatively, a description of the extrema of the Hamilton's action (14) can be obtained 
using the Eisenhart metric (see Eisenhart, 1929) on an enlarged LSF space-time manifold (given by $\left\{q^{0} \equiv t, q^{1}, \ldots, q^{N}\right\}$ plus one real coordinate $q^{N+1}$ ), whose arc-length is

$$
d s^{2}=-2 V(q)\left(d q^{0}\right)^{2}+a_{i j} d q^{i} d q^{j}+2 d q^{0} d q^{N+1} .
$$

The manifold has a Lorentzian structure (Pettini, 2007) and the dynamical trajectories are those geode-sics satisfying the condition $d s^{2}=$ $C d t^{2}$, where $C$ is a positive constant. In this geometrical framework, the instability of the trajectories is the instability of the geodesics, and it is completely determined by the curvature properties of the LSF-manifold $\boldsymbol{\Sigma}$ according to the Jacobi equation of geodesic deviation (see Ivancevic, 2006b, 2007a)

$$
\frac{D^{2} J^{i}}{d s^{2}}+R_{j k m}^{i} \frac{d q^{j}}{d s} J^{k} \frac{d q^{m}}{d s}=0,
$$

whose solution $J$, usually called Jacobi variation field, locally measures the distance between nearby geodesics; $D / d s$ stands for the covariant derivative along a geodesic and $R_{j k m}^{i}$ are the components of the Riemann curvature tensor of the LSF-manifold $\boldsymbol{\Sigma}$.

Using the Eisenhart metric (17), the relevant part of the Jacobi equation (18) is given by the tangent dynamics equation (Casetti et al, 1996; Caiani et al, 1997)

$$
\frac{d^{2} J^{i}}{d t^{2}}+R_{0 k 0}^{i} J^{k}=0, \quad(i=1, \ldots, N),
$$

where the only non-vanishing components of the curvature tensor of the LSF-manifold $\boldsymbol{\Sigma}$ are

$$
R^{i}{ }_{0 k 0}=\partial^{2} V / \partial q^{i} \partial q^{j} .
$$

The tangent dynamics equation (19) is commonly used to define Lyapunov exponents in dynamical systems given by the Riemannian action (14) and Hamiltonian (15), using the formula (Casetti et al, 2000)

$$
\begin{aligned}
\lambda_{1} & =\lim _{t \rightarrow \infty} 1 / 2 t \log \left(\Sigma _ { i = 1 } ^ { N } \left[J_{i}^{2}(t)\right.\right. \\
& \left.\left.+J_{i}^{2}(t)\right] / \Sigma_{i=1}^{N}\left[J_{i}^{2}(0)+J_{i}^{2}(0)\right]\right) .
\end{aligned}
$$

Lyapunov exponents measure the strength of dynamical chaos.

Now, to relate these results to topological phase transitions within the LSF-manifold $\boldsymbol{\Sigma}$, recall that any two high-dimensional manifolds $\Sigma_{v}$ and $\Sigma_{v^{\prime}}$ have the same topology if they can be continuously and differentiably deformed into one another, that is if they are diffeomorphic. Thus by topology change the 'loss of diffeomorphicity' is meant (Pettini, 2007). In this respect, the so-called topological theorem (Franzosi and Pettinni, 2004) says that non-analyticity is the 'shadow' of a more fundamental phenomenon occurring in the system's configuration manifold (in our case the LSF-manifold): a topology change within the family of equipotential hypersurfaces

$\Sigma_{v}=\left\{\left(q^{1}, \ldots, q^{N}\right) \in \mathbb{R}^{N} \mid V\left(q^{1}, \ldots, q^{N}\right)=v\right\}$,

where $V$ and $q^{i}$ are the microscopic interaction potential and coordinates respectively. This topological approach to PTs stems from the numerical study of the dynamical counterpart of phase transitions, and precisely from the observation of discontinuous or cuspy patterns displayed by the largest Lyapunov exponent $\lambda_{1}$ at the transition energy (Casetti et al, 2000). Lyapunov exponents cannot be measured in laboratory experiments, at variance with thermodynamic observables, thus, being genuine dynamical observables they are only be estimated in numerical simulations of the microscopic dy- 
namics. If there are critical points of $V$ in configuration space, that is points $q_{c}=\left[\bar{q}_{1}, \ldots, \bar{q}_{N}\right]$ such that $\left.\nabla V(q)\right|_{q=q_{c}}=0$, according to the Morse Lemma (see e.g., Hirsch, 1976), in the neighborhood of any critical point $q_{c}$ there always exists a coordinate system $\tilde{q}(t)=\left[\tilde{q}^{1}(t), . ., \tilde{q}^{N}(t)\right]$ for which

$V(\tilde{q})=V\left(q_{c}\right)-\tilde{q}_{1}^{2}-\cdots-\tilde{q}_{k}^{2}+\tilde{q}_{k+1}^{2}+\cdots+\tilde{q}_{N}^{2}$,

where $k$ is the index of the critical point, i.e., the number of negative eigenvalues of the Hessian of the potential energy $V$. In the neighborhood of a critical point of the LSF-manifold $\Sigma$, (21) yields

$$
\partial^{2} V / \partial q^{i} \partial q^{j}= \pm \delta_{i j}
$$

which gives $k$ unstable directions which contribute to the exponential growth of the norm of the tangent vector $J$ (Casetti et all, 2000).

This means that the strength of dynamical chaos within the individual's LSF-manifold $\boldsymbol{\Sigma}$, measured by the largest Lyapunov exponent $\lambda_{1}$ given by (20), is affected by the existence of critical points $q_{c}$ of the potential energy $V(q)$. However, as $V(q)$ is bounded below, it is a good Morse function, with no vanishing eigenvalues of its Hessian matrix. According to Morse theory, the existence of critical points of $V$ is associated with topology changes of the hypersurfaces $\left\{\Sigma_{v}\right\}_{v \in \mathbb{R}}$.

More precisely, let $V_{N}\left(q_{1}, \ldots, q_{N}\right): R^{N} \rightarrow$ $R$, be a smooth, bounded from below, finiterange and confining potentia 2 . Denote by $\Sigma_{v}=$ $V^{-1}(v), v \in R$, its level sets, or equipotential hypersurfaces, in the LSF-manifold $\boldsymbol{\Sigma}$. Then

\footnotetext{
${ }^{2}$ These requirements for $V$ are fulfilled by standard interatomic and intermolecular interaction potentials, as well as by classical spin potentials.
}

let $\bar{v}=v / N$ be the potential energy per degree of freedom. If there exists $N_{0}$, and if for any pair of values $\bar{v}$ and $\bar{v}^{\prime}$ belonging to a given interval $I_{\bar{v}}=\left[\bar{v}_{0}, \bar{v}_{1}\right]$ and for any $N>N_{0}$ then the sequence of the Helmoltz free energies $\left\{F_{N}(\beta)\right\}_{N \in \mathbb{N}}-$ where $\beta=1 / T$ ( $T$ is the temperature) and $\beta \in I_{\beta}=\left(\beta\left(\bar{v}_{0}\right), \beta\left(\bar{v}_{1}\right)\right)-$ is uniformly convergent at least in $C^{2}\left(I_{\beta}\right)$ [the space of twice differentiable functions in the interval $\left.I_{\beta}\right]$, so that $\lim _{N \rightarrow \infty} F_{N} \in C^{2}\left(I_{\beta}\right)$ and neither first nor second order phase transitions can occur in the (inverse) temperature interval $\left(\beta\left(\bar{v}_{0}\right), \beta\left(\bar{v}_{1}\right)\right)$, where the inverse temperature is defined as (Pettini, 2007)

$$
\begin{aligned}
& \beta(\bar{v})=\partial S_{N}^{(-)}(\bar{v}) / \partial \bar{v}, \quad \text { while } \\
& S_{N}^{(-)}(\bar{v})=N^{-1} \log \int_{V(q) \leq \bar{v} N} d^{N} q
\end{aligned}
$$

is one of the possible definitions of the microcanonical configurational entropy. The intensive variable $\bar{v}$ has been introduced to ease the comparison between quantities computed at different $N$-values.

This theorem means that a topology change of the $\left\{\Sigma_{v}\right\}_{v \in \mathbb{R}}$ at some $v_{c}$ is a necessary condition for a phase transition to take place at the corresponding energy value. The topology changes implied here are those described within the framework of Morse theory through 'attachment of handles' to the LSF-manifold $\boldsymbol{\Sigma}$ (Hirsch, 1976).

In the LSF path-integral language, we can say that suitable topology changes of equipotential submanifolds of the individual's LSFmanifold $\boldsymbol{\Sigma}$ can entail thermodynamic-like phase transitions, according to the general formula:

$$
\begin{aligned}
& \langle\text { phase out }| \text { phase in }\rangle:= \\
& \oint_{\text {topology-change }} \mathcal{D}[w \Phi] \mathrm{e}^{\mathrm{i} S[\Phi]} .
\end{aligned}
$$


The statistical behavior of the LSF-(loco)motion system (14) with the standard Hamiltonian (15) is encompassed, in the canonical ensemble, by its partition function, given by the phasespace path integral (Ivancevic, 2007a, 2008a)

$Z_{N}=\oiint_{\text {top-ch }} \mathcal{D}[p] \mathcal{D}[q] \exp \left\{\mathrm{i} \int_{t}^{t^{\prime}}[p \dot{q}-H(p, q)] d \tau\right\}$

where we have used the shorthand notation

$$
\oiint_{\text {top }-\mathrm{ch}} \mathcal{D}[p] \mathcal{D}[q] \equiv \int \prod_{\tau} \frac{d q(\tau) d p(\tau)}{2 \pi} .
$$

The phase-space path integral (22) can be calculated as the partition function (Franzosi et al, 2000)

$$
\begin{aligned}
& Z_{N}(\beta)=\int \prod_{i=1}^{N} d p_{i} d q^{i} \mathrm{e}^{-\beta H(p, q)} \\
& =\left(\frac{\pi}{\beta}\right)^{\frac{N}{2}} \int \prod_{i=1}^{N} d q^{i} \mathrm{e}^{-\beta V(q)} \\
& =\left(\frac{\pi}{\beta}\right)^{\frac{N}{2}} \int_{0}^{\infty} d v \mathrm{e}^{-\beta v} \int_{\Sigma_{v}} \frac{d \sigma}{\|\nabla V\|^{2}},
\end{aligned}
$$

where the last term is written using the socalled co-area formula (Federer, 1969), and $v$ labels the equipotential hypersurfaces $\Sigma_{v}$ of the LSF-manifold $\boldsymbol{\Sigma}$,

$\Sigma_{v}=\left\{\left(q^{1}, \ldots, q^{N}\right) \in \mathbb{R}^{N} \mid V\left(q^{1}, \ldots, q^{N}\right)=v\right\}$.

Equation (23) shows that the relevant statistical information is contained in the canonical configurational partition function

$$
Z_{N}^{C}=\int \prod d q^{i} V(q) \mathrm{e}^{-\beta V(q)} .
$$

Note that $Z_{N}^{C}$ is decomposed, in the last term of (23), into an infinite summation of geometric integrals,

$$
\int_{\Sigma_{v}} d \sigma /\|\nabla V\|
$$

defined on the $\left\{\Sigma_{v}\right\}_{v \in \mathbb{R}}$. Once the microscopic interaction potential $V(q)$ is given, the configu,ration space of the system is automatically foliated into the family $\left\{\Sigma_{v}\right\}_{v \in \mathbb{R}}$ of these equipotential hypersurfaces. Now, from standard statistical mechanical arguments we know that, at any given value of the inverse temperature $\beta$, the larger the number $N$, the closer to $\Sigma_{v} \equiv$ $\Sigma_{u_{\beta}}$ are the microstates that significantly contribute to the averages, computed through $Z_{N}(\beta)$, of thermodynamic observables. The hypersurface $\Sigma_{u_{\beta}}$ is the one associated with

$$
u_{\beta}=\left(Z_{N}^{C}\right)^{-1} \int \prod d q^{i} V(q) \mathrm{e}^{-\beta V(q)},
$$

the average potential energy computed at a given $\beta$. Thus, at any $\beta$, if $N$ is very large the effective support of the canonical measure shrinks very close to a single $\Sigma_{v}=\Sigma_{u_{\beta}}$. Hence, the basic origin of a phase transition lies in a suitable topology change of the $\left\{\Sigma_{v}\right\}$, occurring at some $v_{c}$ (Franzosi et al, 2000). This topology change induces the singular behavior of the thermodynamic observables at a phase transition. It is conjectured that the counterpart of a phase transition is a breaking of diffeomorphicity among the surfaces $\Sigma_{v}$, it is appropriate to choose a diffeomorphism invariant to probe if and how the topology of the $\Sigma_{v}$ changes as a function of $v$. Fortunately, such a topological invariant exists, the Euler characteristic of the LSF-manifold $\Sigma$, defined by (Ivancevic, 2007a)

$$
\chi(\Sigma)=\sum_{k=0}^{N}(-1)^{k} b_{k}(\Sigma),
$$


where the Betti numbers $b_{k}(\Sigma)$ are diffeomorphism invariants 3 This homological formula can be simplified by the use of the Gauss-Bonnet Hopf theorem, that relates $\chi(\Sigma)$ with the total Gauss-Kronecker curvature $K_{G}$ of the LSFmanifold $\boldsymbol{\Sigma}$

$$
\chi(\Sigma)=\int_{\Sigma} K_{G} d \sigma
$$

where

$$
d \sigma=\sqrt{\operatorname{det}(a)} d x^{1} d x^{2} \cdots d x^{n}
$$

is the invariant volume measure of the LSFmanifold $\boldsymbol{\Sigma}$ and $a$ is the determinant of the LSF metric tensor $a_{i j}$ (Ivancevic, 2008a).

The domain of validity of the 'quantum' is not restricted to the microscopic world (Umezaw 1993). There are macroscopic features of classically behaving systems, which cannot be explained without recourse to the quantum dynamics. This field theoretic model leads to the view of the phase transition as a condensation that is comparable to the formation of fog and rain drops from water vapor, and that might serve to model both the gamma and beta phase transitions. According to such a model, the production of activity with long-range correlation in the brain takes place through the mechanism of spontaneous breakdown of symmetry (SBS), which has for decades been shown to describe long-range correlation in condensed matter physics. The adoption of such a field theoretic approach enables modelling of the whole cerebral hemisphere and its hierarchy of components down to the atomic level as a fully integrated macroscopic quantum system, namely

\footnotetext{
${ }^{3}$ The Betti numbers $b_{k}$ are the dimensions of the de Rham's cohomology vector spaces $H^{k}(\Sigma ; \mathbb{R}$ ) (therefore the $b_{k}$ are integers).
}

as a macroscopic system which is a quantum system not in the trivial sense that it is made, like all existing matter, by quantum components such as atoms and molecules, but in the sense that some of its macroscopic properties can best be described with recourse to quantum dynamics (see Freeman and Vitiello, 2006 and references therein).

Phase transitions can also be associated with autonomous robot competence levels, as informal specifications of desired classes of behaviors for robots over all environments they will encounter, as described by Brooks' subsumption architecture approach. The distributed network of augmented finite-state machines can exist in different phases or modalities of their state-space variables, which determine the systems intrinsic behavior. The phase transition represented by this approach is triggered by either internal (a set-point) or external (a command) control stimuli, such as a command to transition from a sleep mode to awake mode, or walking to running.

\section{Joint Action of Several Agents}

In this section we propose an LSF-based model of the joint action of two or more actors, where actors can be both humans and robots. This joint action takes place in the joint LSF manifold $\Sigma_{J}$, composed of individual LSF manifolds $\Sigma_{\alpha}, \Sigma_{\beta}, \ldots$. It has a sophisticated geometrical and dynamical structure as follows.

To model the dynamics of the two-actor $\mathrm{co}^{-}$ action, we propose to associate each of the actors with a set of their own time dependent trajectories, which constitutes an $n$-dimensional Riemannian LSF-manifold, $\Sigma_{\alpha}=\left\{x^{i}\left(t_{i}\right)\right\}$ and 
$\Sigma_{\beta}=\left\{y^{j}\left(t_{j}\right)\right\}$, respectively. Their associated tangent bundles contain their individual $n \mathrm{D}$ (loco)motion velocities, $T \Sigma_{\alpha}=\left\{\dot{x}^{i}\left(t_{i}\right)=d x^{i} / d t_{i}\right\}$ and $T \Sigma_{\beta}=\left\{\dot{y}^{j}\left(t_{j}\right)=d y^{j} / d t_{j}\right\}$. Further, following the general LSF-formalism, outlined in the introduction, we use the modelling machinery consisting of: (i) Adaptive joint action at the top-master level, describing the externallyappearing deterministic, continuous and smooth dynamics, and (ii) Corresponding adaptive path integral (30) at the bottom-slave level, describing a wildly fluctuating dynamics including both continuous trajectories and Markov chains. This lower-level joint dynamics can be further discretized into a partition function of the corresponding statistical dynamics.

The smooth joint action with two terms, representing cognitive/motivational potential energy and physical kinetic energy, is formally given by:

$$
\begin{aligned}
& A\left[x, y ; t_{i}, t_{j}\right]= \\
& \frac{1}{2} \int_{t_{i}} \int_{t_{j}} \alpha_{i} \beta_{j} \delta\left(I_{i j}^{2}\right) \dot{x}^{i}\left(t_{i}\right) \dot{y}^{j}\left(t_{j}\right) d t_{i} d t_{j} \\
& +\frac{1}{2} \int_{t} g_{i j} \dot{x}^{i}(t) \dot{x}^{j}(t) d t, \\
& \text { with } \quad I_{i j}^{2}=\left[x^{i}\left(t_{i}\right)-y^{j}\left(t_{j}\right)\right]^{2}, \\
& \text { where } \quad I N \leq t_{i}, t_{j}, t \leq O U T .
\end{aligned}
$$

The first term in (26) represents potential energy of the cognitive/motivational interaction between the two agents $\alpha_{i}$ and $\beta_{j}$ 近 It is a double integral over a delta function of the square of interval $I^{2}$ between two points on the paths in their Life-Spaces; thus, interaction occurs only when this interval, representing the motivational cognitive distance between the two

\footnotetext{
${ }^{4}$ Although, formally, this term contains cognitive velocities, it still represents 'potential energy' from the physical point of view.
}

agents, vanishes. Note that the cognitive (loco) motions of the two agents $\alpha_{i}\left[x^{i}\left(t_{i}\right)\right]$ and $\beta_{j}\left[y^{j}\left(t_{j}\right)\right]$, ggenerally occur at different times $t_{i}$ and $t_{j}$ unless $t_{i}=t_{j}$, when cognitive synchronization occurs.

The second term in (26) represents kinetic energy of the physical interaction. Namely, when the cognitive synchronization in the first term takes place, the second term of physical kinetic energy is activated in the common manifold, which is one of the agents' Life Spaces, say $\Sigma_{\alpha}=\left\{x^{i}\left(t_{i}\right)\right\}$.

Conversely, if we have a need to represent coaction of three actors, say $\alpha_{i}, \beta_{j}$ and $\gamma_{k}$ (e.g., $\alpha_{i}$ in charge of acceleration, $\beta_{j}$ - deceleration and $\gamma_{k}$ - steering), we can associate each of them with an $n \mathrm{D}$ Riemannian Life-Space manifold, $\Sigma_{\alpha}=\left\{x^{i}\left(t_{i}\right)\right\}, \Sigma_{\beta}=\left\{y^{j}\left(t_{j}\right)\right\}$, and $\Sigma_{\gamma}=$ $\left\{z^{k}\left(t_{k}\right)\right\}$, respectively, with the corresponding tangent bundles containing their individual (loco) motion velocities, $T \Sigma_{\alpha}=\left\{\dot{x}^{i}\left(t_{i}\right)=d x^{i} / d t_{i}\right\}$, $T \Sigma_{\beta}=\left\{\dot{y}^{j}\left(t_{j}\right)=d y^{j} / d t_{j}\right\}$ and $T \Sigma_{\gamma}=\left\{\dot{z}^{k}\left(t_{k}\right)\right.$ $\left.=d z^{k} / d t_{k}\right\}$. Then, instead of (26) we have

$$
\begin{aligned}
& A\left[t_{i}, t_{j}, t_{k} ; t\right]= \\
& \frac{1}{2} \int_{t_{i}} \int_{t_{j}} \int_{t_{k}} \alpha_{i}\left(t_{i}\right) \beta_{j}\left(t_{j}\right) \gamma_{k}\left(t_{k}\right) \times \\
& \delta\left(I_{i j k}^{2}\right) \dot{x}^{i}\left(t_{i}\right) \dot{y}^{j}\left(t_{j}\right) \dot{z}^{k}\left(t_{k}\right) d t_{i} d t_{j} d t_{k} \\
& +\frac{1}{2} \int_{t} W_{r s}^{M}(t, q, \dot{q}) \dot{q}^{r} \dot{q}^{s} d t,
\end{aligned}
$$

where $I N \leq t_{i}, t_{j}, t_{k}, t \leq O U T, \quad$ with $I_{i j k}^{2}=\left[x^{i}\left(t_{i}\right)-y^{j}\left(t_{j}\right)\right]^{2}+\left[y^{j}\left(t_{j}\right)-z^{k}\left(t_{k}\right)\right]^{2}$ $+\left[z^{k}\left(t_{k}\right)-x^{i}\left(t_{i}\right)\right]^{2}$,

Due to an intrinsic chaotic coupling, the three-actor (or, $n$-actor, $n>3$ ) joint action (27) has a considerably more complicated geometrical structure then the bilateral co-action 
(26) 5 It actually happens in the common $3 n \mathrm{D}$ Finsler manifold $\Sigma_{J}=\Sigma_{\alpha} \cup \Sigma_{\beta} \cup \Sigma_{\gamma}$, parameterized by the local joint coordinates dependent on the common time $t$. That is, $\Sigma_{J}=$ $\left\{q^{r}(t), r=1, \ldots, 3 n\right\}$. Geometry of the joint manifold $\Sigma_{J}$ is defined by the Finsler metric function $d s=F\left(q^{r}, d q^{r}\right)$, defined by

$$
F^{2}(q, \dot{q})=g_{r s}(q, \dot{q}) \dot{q}^{r} \dot{q}^{s}
$$

and the Finsler tensor $C_{r s t}(q, \dot{q})$, defined by (Ivancevic, 2007a)

$$
C_{r s t}(q, \dot{q})=\frac{1}{4} \frac{\partial^{3} F^{2}(q, \dot{q})}{\partial \dot{q}^{r} \partial \dot{q}^{s} \partial \dot{q}^{t}}=\frac{1}{2} \frac{\partial g_{r s}}{\partial \dot{q}^{r} \partial \dot{q}^{s}} .
$$

From the Finsler definitions (28)-(29), it follows that the partial interaction manifolds, $\Sigma_{\alpha} \cup$ $\Sigma_{\beta}, \Sigma_{\beta} \cup \Sigma_{y}$ and $\Sigma_{\alpha} \cup \Sigma_{y}$, have Riemannian structures with the corresponding interaction kinetic energies,

$$
\begin{aligned}
T_{\alpha \beta} & =\frac{1}{2} g_{i j} \dot{x}^{i} \dot{y}^{j}, \quad T_{\alpha \gamma}=\frac{1}{2} g_{i k} \dot{x}^{i} \dot{z}^{k}, \\
T_{\beta \gamma} & =\frac{1}{2} g_{j k} \dot{y}^{j} \dot{z}^{k} .
\end{aligned}
$$

At the slave LSF-level, the adaptive path integral, representing an $\infty$-dimensional neural network, corresponding to the adaptive bilateral joint action (26), reads

$$
\langle O U T \mid I N\rangle:=£ \mathcal{D}[w, x, y] \mathrm{e}^{\mathrm{i} A\left[x, y ; t_{i}, t_{j}\right]},
$$

where the Lebesgue integration is performed over all continuous paths $x^{i}=x^{i}\left(t_{i}\right)$ and $y^{j}=$ $y^{j}\left(t_{j}\right)$, while summation is performed over all associated discrete Markov fluctuations and jumps. The symbolic differential in the path

\footnotetext{
${ }^{5}$ Recall that the necessary condition for chaos in continuous temporal or spatio-temporal systems is to have three variables with nonlinear couplings between them.
}

integral (30) represents an adaptive path measure, defined as a weighted product

$$
\begin{array}{r}
\mathcal{D}[w, x, y]=\lim _{N \rightarrow \infty} \prod_{s=1}^{N} w_{i j}^{s} d x^{i} d y^{j}, \\
(i, j=1, \ldots, n) .
\end{array}
$$

Similarly, in case of the triple joint action, the adaptive path integral reads,

$$
\langle O U T \mid I N\rangle:=£ \mathcal{D}[w ; x, y, z ; q] \mathrm{e}^{\mathrm{i} A\left[t_{i}, t_{j}, t_{k} ; t\right]},
$$

with the adaptive path measure defined by

$$
\begin{gathered}
\mathcal{D}[w ; x, y, z ; q]=\lim _{N \rightarrow \infty} \prod_{S=1}^{N} w_{i j k r}^{S} d x^{i} d y^{j} d z^{k} d q^{r} \\
(i, j, k=1, \ldots, n ; r=1, \ldots, 3 n) .
\end{gathered}
$$

The proposed path integral approach represents a new family of more expansive functionrepresentation methods, which is now capable of representing input/output behavior of more than one actor. However, as we add the second and subsequent actors to the model, the requirements for the rigorous geometrical representations of their respective LSFs become nontrivial. For a single actor or a two-actor co-action the Riemannian geometry was sufficient, but it becomes insufficient for modelling the $n$-actor (with $n \geq 3$ ) joint action, due to an intrinsic chaotic coupling between the individual actors' LSFs. To model an $n$-actor joint LSF, we have to use the Finsler geometry, which is a generalization of the Riemannian one. This progression may seem trivial, both from standard psychological point of view, and from computational point of view, but it is not trivial from the geometrical perspective.

Our extended LSF formalism is closely related to the Haken-Kelso-Bunz (HKB) model 
of self-organization in the human motor system (Haken et al, 1985; Kelso, 1995), including: multi-stability, phase transitions and hysteresis effects, presenting a contrary view to the purely feedback driven neural systems. HKB uses the concepts of synergetics (order parameters, control parameters, instability, etc) and the mathematical tools of nonlinearly coupled (nonlinear) dynamical systems to account for self-organized behavior both at the cooperative, coordinative level and at the level of the individual coordinating elements. The HKB model stands as a building block upon which numerous extensions and elaborations have been constructed. In particular, it has been possible to derive it from a realistic model of the cortical sheet in which neural areas undergo a reorganization that is mediated by intra- and intercortical connections. Also, the HKB model describes phase transitions ('switches') in coordinated human movement as follows: (i) when the agent begins in the anti-phase mode and speed of movement is increased, a spontaneous switch to symmetrical, in-phase movement occurs; (ii) this transition happens swiftly at a certain critical frequency; (iii) after the switch has occurred and the movement rate is now decreased the subject remains in the symmetrical mode, i.e. she does not switch back; and (iv) no such transitions occur if the subject begins with symmetrical, in-phase movements. The HKB dynamics of the order parameter relative phase as is given by a nonlinear first-order ODE:

$$
\dot{\phi}=\left(\alpha+2 \beta r^{2}\right) \sin \phi-\beta r^{2} \sin 2 \phi,
$$

where $\phi$ is the phase relation (that characterizes the observed patterns of behavior, changes abruptly at the transition and is only weakly dependent on parameters outside the phase transition), $r$ is the oscillator amplitude, while $\alpha, \beta$ are coupling parameters (from which the critical frequency where the phase transition occurs can be calculated).

From a quantum perspective, closely related to the LSF model are the recent developments of Hong and Newell (2008a, 2008b) in motor control that deal with feedback information and environmental uncertainty. The probabilistic nature of human action can be characterized by entropies at the level of the organism, task, and environment. Systematic changes in motor adaptation are characterized as task-organism and environment-organism tradeoffs in entropy. Such compensatory adaptations lead to a view of goal-directed motor control as the product of an underlying conservation of entropy across the task-organism-environment system. The conservation of entropy supports the view that context dependent adaptations in human goaldirected action are guided fundamentally by natural law and provides a novel means of examining human motor behavior. This is fundamentally related to the Heisenberg uncertainty principle and further support the argument for the primacy of a probabilistic approach toward the study of bio-psychological systems. 


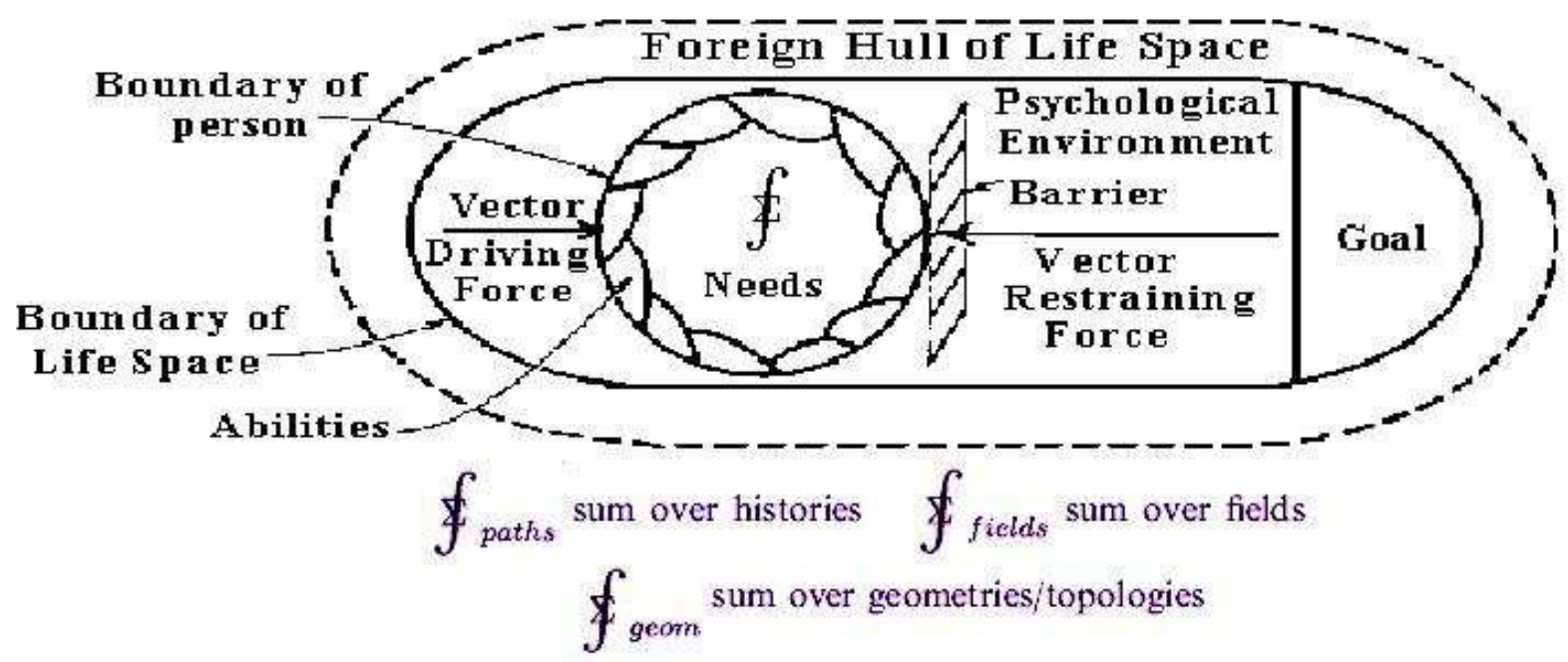

Figure 2: Diagram of the life space foam: classical representation of Lewinian life space, with an adaptive path integral $\mathcal{f}$ (denoting integration over continuous paths and summation over discrete Markov jumps) acting inside it and generating microscopic fluctuation dynamics. 


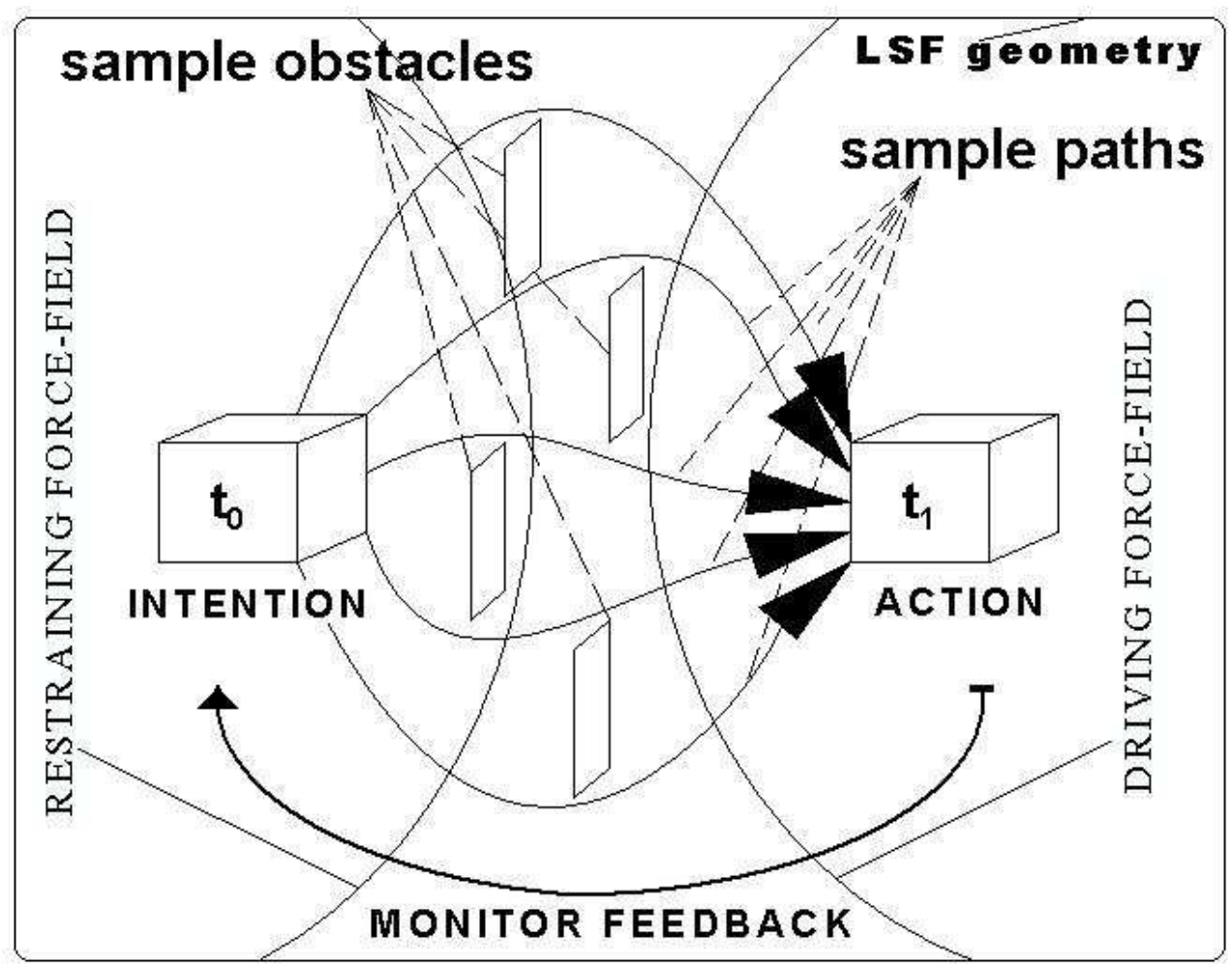

Figure 3: Transition-propagator corresponding to each of the motivational phases $\{\mathcal{F}, \mathcal{I}, \mathcal{M}, \mathcal{T}\}$, consisting of an ensemble of feedforward paths propagating through the 'wood of obstacles'. The paths affected by driving and restraining force-fields, as well as by the local LSF-geometry. Transition goes from Intention, occurring at a sample time instant $t_{0}$, to Action, occurring at some later time $t_{1}$. Each propagator is controlled by its own Monitor feedback. 


$$
\begin{aligned}
& A=\frac{1}{2} \int_{t_{i}} \int_{t_{j}} \alpha_{i} \beta_{j} \delta\left(I_{i j}^{2}\right) \dot{x}_{\text {two timescales }}^{\dot{i}^{i}}\left(t_{i}\right) \dot{y}^{j}\left(t_{j}\right) d t_{i} d t_{j} \\
& +\frac{1}{2} \int_{t} \begin{array}{c}
\text { physical kinetic energy } \\
g_{i j} \dot{x}^{i}(t) \dot{y}^{j}(t) d t \\
\text { one timescale }
\end{array} \\
& \text { with } I_{i j}^{2}=\left[x^{i}\left(t_{i}\right)-y^{j}\left(t_{j}\right)\right]^{2}
\end{aligned}
$$

Figure 4: Feynman action for modelling human joint action, including potential energy (motivational cognition) in two timescales, physical energy in a single timescale (after synchronization has already occurred), and the distance between two agents in the motivational cognition space. 


\section{Conclusion}

General stochastic and quantum dynamics, developed in a framework of Feynman path integrals, have recently been applied by Ivancevic and Aidman (2007) to Lewinian field-theoretic psychodynamics, resulting in the development of a new concept of Life-Space Foam (LSF) as a natural medium for motivational (MD) and cognitive (CD) psychodynamics. According to the LSF-formalism, the classic Lewinian life space can be macroscopically represented as a smooth manifold with steady force-fields and behavioral paths, while at the microscopic level it is more realistically represented as a collection of wildly fluctuating force-fields, (loco)motion paths and local geometries (and topologies with holes). This paper extends the LSFmodel to incorporate the notion of phase transitions and complements it with embedded geometrical chaos. As a result, the extended LSFmodel is able to rigorously represent co-action by two or more human-like agents in the common LSF-manifold. The extended LSF-model is also related to the HKB-model of self-organization in the human motor system, presenting a contrary view to the purely feedback driven neural systems, as well as Hong-Newell entropyapproach to adaptation in human goal-directed motor control.

\section{References}

Amari S. Dynamics of pattern formation in lateral-inhibition type neural fields. Biol $\mathrm{Cy}-$ bern 1977;27:77-87.

Bernstein NA, Latash ML and Turvey MT (Eds). Dexterity and its development. Hillsdale NJ England: Lawrence Erlbaum Associates
1996.

Bernstein NA. Some emergent problems of the regulation of motor acts. In: Whiting $\mathrm{H}$ (ed) Human Motor Actions: Bernstein Reassessed 343-358. North Holland, Amsterdam 1982.

Bernstein NA. The Coordination and Regulation of Movements. Pergamon London 1967.

Broadbent DE. Perception and communications. Pergamon Press, London 1958.

Brooks RA. A Robust Layered Control System for a Mobile Robot. IEEE Trans Rob Aut 1986;(2)1:14-23.

Brooks RA. A robot that walks: Emergent behavior form a carefully evolved network. Neu Comp 1989;12:253-262.

Brooks RA. Elephants Don't Play Chess. Rob Aut Sys 1990;6:3-15.

Busemeyer JR, Wang Z and Townsend JT. Quantum dynamics of human decision-making. J Math Psych 2006;50:220-241.

Caiani L, Casetti L, Clementi C and Pettini M. Geometry of Dynamics Lyapunov Exponents and Phase Transitions. Phys Rev Lett 1997;79:4361-4364.

Casetti L, Clementi C and Pettini M. Riemannian theory of Hamiltonian chaos and Lyapunov exponents. Phys Rev E 1996;54:5969.

Casetti L, Pettini M and Cohen EGD. Geometric Approach to Hamiltonian Dynamics and Statistical Mechanics. Phys Rep 2000;337:237341.

Droll, JA, Hayhoe, MM, Triesch, J and Sullivan, BT. Task demands control acquisition and storage of visual information. J Exp Psych: Hum Perc Perf 2005;31:14161438.

Eisenhart LP. Dynamical trajectories and geodesics. Math Ann 1929;30:591-606.

Federer H. Geometric Measure Theory. Springer New York 1969. 
Fogassi L, Ferrari PF, Gesierich B, Rozzi S, Chersi F and Rizzolatti G. Parietal lobe: From action organization to intention understanding. Science 2005;29:662-667.

Franzosi R and Pettini M. Theorem on the origin of Phase Transitions. Phys Rev Lett 2004;92:060601.

Franzosi R, Pettini M, Spinelli L. Topology and phase transitions: a paradigmatic evidence. Phys Rev Lett 2000;84:2774-2777.

Freeman WJ and Vitiello G. Nonlinear brain dynamics as macroscopic manifestation of underlying many-body field dynamics. Phys Life Rev 2006;(3)2:93-118.

Freeman WJ. Mass Action in the Nervous System. Acad Press, New York 1975/2004.

Freeman WJ. Neurodynamics. An Exploration of Mesoscopic Brain Dynamics. Springer, London 2000.

Gardiner C.W. Handbook of Stochastic Methods for Physics Chemistry and Natural Sciences 2nd ed. Springer, New York 1985.

Glimcher PW. Indeterminacy in brain and behaviour. Ann Rev Psych 2005;56:25-56.

Gold M. A Kurt Lewin Reader the Complete Social Scientist. Am Psych Assoc Washington 1999.

Haken H. Advanced Synergetics: Instability Hierarchies of Self-Organizing Systems and Devices (3nd ed). Springer, Berlin 1993.

Haken H. Principles of Brain Functioning: A Synergetic Approach to Brain Activity Behavior and Cognition. Springer, Berlin 1996.

Haken H. Synergetics: An Introduction (3rd ed). Springer, Berlin 1983.

Haken, H, Kelso, JAS and Bunz H. A theoretical model of phase transitions in human hand movements. Biol Cybern 1985;51:347356.
Heermann DW. Computer Simulation Methods in Theoretical Physics (2nd ed). Springer, Berlin 1990.

Hirsch MW. Differential Topology. Springer, New York 1976.

Hong SL, Newell KM. Entropy compensation in human motor adaptation. Chaos

2008b;18(1):013108.

Hong SL, Newell KM. Entropy conservation in the control of human action. Nonl Dyn Psych Life Sci 2008a;12(2):163-190.

Ivancevic T, Jain L, Pattison J and Hariz A. Nonlinear Dynamics and Chaos Methods in Neurodynamics and Complex Data Analysis. Nonl Dyn 2008(in press, on line first, Springer).

Ivancevic $\mathrm{V}$ and Aidman E. Life-space foam: A medium for motivational and cognitive dynamics. Physica A 2007;382:616-630.

Ivancevic V and Ivancevic T. Applied Dif-ferential Geometry: A Modern Introduction. World Scientific, Singapore 2007a.

Ivancevic $\mathrm{V}$ and Ivancevic T. Complex Nonlinearity: Chaos, Phase Transitions, Topology Change and Path Integrals. Springer, Berlin 2008a.

Ivancevic $\mathrm{V}$ and Ivancevic T. Computational Mind: A Complex Dynamics Perspective.

Springer, Berlin 2007c.

Ivancevic $\mathrm{V}$ and Ivancevic T. Geometrical Dynamics of Complex Systems: A Unified Modelling Approach to Physics Control Biomechanics Neurodynamics and Psycho-Socio-Economical Dynamics. Springer, Dordrecht 2006b.

Ivancevic V and Ivancevic T. High-Dimensional Chaotic and Attractor Systems. Springer, Berlin 2006c.

Ivancevic V and Ivancevic T. Natural Biodynamics. World Scientific, Singapore 2006a.

Ivancevic V and Ivancevic T. Neuro-Fuzzy Associative Machinery for Comprehensive Brain 
and Cognition Modelling. Springer, Berlin 2007b. Sebanz N, Bekkering H and Knoblich G.

Ivancevic V and Ivancevic T. Quantum Leap: Joint action: bodies and minds moving together. From Dirac and Feynman Across the Universe Tr Cog Sci 2006;(10)2:70-76.

to Human Body and Mind. World Scientific, Sutton RS and Barto AG. Reinforcement Singapore 2008b. Learning: An Introduction. MIT Press, Cam-

Izhikevich EM and Edelman GM. Large- bridge 1998.

Scale Model of Mammalian Thalamocortical Sys- Todorov E, Jordan MI. Optimal feedback tems. PNAS 2008;105:3593-3598.

control as a theory of motor coordination. Nat

Kelso, JAS. Dynamic Patterns: The Self Neurosci 2002;(5)11:1226-1235.

Organization of Brain and Behavior. MIT Press, Cambridge 1995.

Tognoli, E, Lagarde, J, DeGuzman, GC and

Knill DC, Maloney LT and Trommershauser J. Sensorimotor Processing and Goal-Directed Movement. J Vis 2007;7(5)i:1-2.

Knoblich G and Jordan S. Action coordination in individuals and groups: Learning anticipatory control. J Exp Psych: Learn Mem Cog 2003;29:1006-1016.

Krylov NS. Works on the foundations of statistical mechanics. Princeton Univ Press 1979.

Land, MF and Hayhoe, M. In what ways do eye movements contribute to everyday activities? Vis Res 2001;41:35593565.

Lewin K. Field Theory in Social Science. Univ Chicago Press 1951.

Lewin K. Resolving Social Conflicts and Field Theory in Social Science. Am Psych Assoc Washington 1997.

Newman-Norlund RD, Noordzij ML, Meulenbroek RGJ and Bekkering H. Exploring the brain basis of joint action: Co-ordination of actions goals and intentions. Soc Neurosci

2007;(2)1:48-65.

Pettini M. Geometry and Topology in Hamiltonian Dynamics and Statistical Mechanics.

Springer, New York 2007.

Schöner G. Dynamical Systems Approaches to Cognition. In: Cambridge Handbook of Computational Cognitive Modelling. Cambridge Univ Press 2007. 